\title{
Accurate finite difference beam propagation method for complex integrated optical structures
}

Rasmussen, Thomas; Povlsen, Jørn Hedegaard; Bjarklev, Anders Overgaard

Published in:

I E E E Photonics Technology Letters

Link to article, DOI:

$10.1109 / 68.205631$

Publication date:

1993

Document Version

Publisher's PDF, also known as Version of record

Link back to DTU Orbit

Citation (APA):

Rasmussen, T., Povlsen, J. H., \& Bjarklev, A. O. (1993). Accurate finite difference beam propagation method for complex integrated optical structures. I E E E Photonics Technology Letters, 5(3), 339-342.

https://doi.org/10.1109/68.205631

\section{General rights}

Copyright and moral rights for the publications made accessible in the public portal are retained by the authors and/or other copyright owners and it is a condition of accessing publications that users recognise and abide by the legal requirements associated with these rights.

- Users may download and print one copy of any publication from the public portal for the purpose of private study or research.

- You may not further distribute the material or use it for any profit-making activity or commercial gain

- You may freely distribute the URL identifying the publication in the public portal 
device length of an air-core ARROW is around $50 \mathrm{~mm}$ (see [3]) thickness, tolerances up to $+/-18 \mathrm{~nm}$ and $+/-16 \mathrm{~nm}$ are allowed.

\section{CONCLUSIONS}

We have shown that the polarization-discrimination performance of an ARROW can be improved considerably by the addition of thin metal layers placed outside the interference cladding layers. Such a metal-clad ARROW polarizer has the following crucial advantages: larger fabrication tolerances than required in conventional waveguide optics, a higher discrimination ratio between the polarization states than in conventional ARROW's, and nearly equal polarization discrimination for the TE field as well as for the TM field. The robustness and the large fabrication tolerances with regard to every parameter of the configuration should encourage the experimental verification of our results.

\section{REFERENCES}

[1] M. A. Duguay, Y. Kokubun, T. L. Koch, and L. Pfeifer, "Antiresonant reflecting optical waveguide in $\mathrm{SiO}_{2}$-Si multilayer structures," Appl. Phys. Lett., vol. 49, pp. 13-15, 1986.

[2] T. Baba and Y. Kokubun, "Dispersion and radiation loss charac- teristics of Antiresonant reflecting optical waveguides-numerical results and analytical expressions," IEEE J. Quantum Electron., vol. QE-28, pp. 1689-1700, 1992.

[3] M. Cantin, C. Carignan, R. Cote, M. A. Duguay, R. Larose, P. LeBel, and F. Quellette, "Remotely switched hollow-core antiresonant reflecting optical waveguide," Opt. Lett., vol. 16, pp. 1738-1740, 1991.

[4] M. Mann, U. Trutschel, C. Waechter, L. Leine, and F. Lederer, "Directional coupler based on an antiresonant reflecting optical waveguide," Opt. Lett., vol. 16, pp. 805-807, 1991.

[5] M. N. Zervas, "Surface plasmon-polariton fiber-optics polarizers using thin chromium films," IEEE Photon. Technol. Lett., vol. 2, pp. $597-599,1990$

[6] K. H. Rollke, W. Sohler, "Metal-clad waveguide as cutoff polarizer for integrated optics," IEEE J. Quantum Electron., vol. QE-16, pp. $141-145,1977$.

[7] D. L. Veasey and D. R. Larson, "In-situ optimization of coupling between semiconductor claddings and dielectric waveguides," $J$. Appl. Phys., vol. 68, pp. 3753-3755, 1990.

[8] K. Thyagarajan, S. Diggavi, and A. K. Ghatak, "Integrated-optic polarization-splitting directional coupler," Opt. Lett., vol. 14, pp. 1333-1335, 1989.

[9] W. Ehrfeld and D. Münchmeyer, "Three-dimensional microfabrication using synchrotron radiation," Nucl. Instruments Method Phys. Res., vol. A303, pp. 523-531, 1991.

[10] M. Born, E. Wolf, Principles of Optics. Englewood Cliffs, NJ: Pergamon, 1984 , p. 60 , equations (49), (50).

[11] E. D. Palik, Handbook of Optical Constants of Solids. New York: Academic, 1985.

\title{
Accurate Finite Difference Beam Propagation Method for Complex Integrated Optical Structures
}

\author{
Thomas Rasmussen, J $\varnothing$ rn Hedegaard Povlsen, and Anders Bjarklev
}

\begin{abstract}
A simple and effective finite difference beam propagation method in a $z$-varying nonuniform mesh is developed. The accuracy and computation time for this method are compared with a standard finite difference method both for 3D and 2D version of the methods.
\end{abstract}

\section{INTRODUCTION}

$\mathrm{D}$ URING the last two decades various numerical method for the investigation of integrated optical structures have been developed. Until lately the most popular method has been the fourier beam propagation method (FBPM) [1] but also different finite difference

Manuscript received October 28, 1992; revised December, 14, 1992. This work was supported by the Danish Technical Research Council and by the National Agency of Industry and Trade, Denmark.

The authors are with the Center for Broadband Telecommunications, Electromagnetics Institute, Technical University of Denmark, DK-2800

Lyngby, Denmark.

IEEE Log Number 9207613 beam propagation methods (FDBPM) have been presented [2]-[4]. However, for large and/or complex integrated optical structures these methods are all time consuming to be accurate.

It has been the purpose of our work to develop a method that enables us to investigate large and complex integrated optical structures accurately without using a long computation time. We have chosen to use a FDBPM due to the simplicity, stability, and independence of computational mesh. Furthermore we use a nonuniform mesh that is finest near the waveguide cores and gradually gets harsher when moving away from the cores. The mesh point spacing is chosen to be minimum at the discontinuities in the refractive index [5]. We show how the mesh may be automatically calculated with very little extra computation effort for step index waveguides. By incorporating an interpolation procedure in the step-algorithm the calculations become dependent on the actual mesh only. This is important for instance in the case of one

$1041-1135 / 93 \$ 03.00$ (c) 1993 IEEE 
waveguide branching into two waveguides since the number of mesh-points will change from step to step. To compare the accuracy and computation time for our method with a standard FDBPM (in a uniform mesh) we have calculated the coupling length of a $3 \mathrm{~dB}$ coupler with curved sections at each end. We did also calculate the coupling length with 2D-versions of the two methods where the original 3D waveguide problem was reduced by using the effective index method [6] to estimate the error committed by doing this.

\section{THEORY}

In the following $x$ and $y$ denote transversal coordinates while $z$ denotes the longitudinal coordinate. It is assumed that all waveguiding structures are weakly guiding so that we may consider the transversely and vertically polarized modes as degenerate. The scalar electric field is written as $E(x, y, z)=\Psi(x, y, z) \cdot \exp \left(j\left(\omega t-n_{0} k z\right)\right)$ where $\omega$ is the angular frequency, $t$ is the time, $k$ the wave number of vacuum, $j$ is the imaginary unit and $n_{0}$ is the refractive index of the medium surrounding the waveguide cores. The Fresnel equation is transformed to the following set of difference equations using Crank-Nicolson's finite differences for a nonuniform but $z$-invariant mesh $\left(x_{i}, y_{j}\right)$

$$
\begin{aligned}
& -j \frac{\Delta z}{2 n_{0} k \Delta x_{i}\left(\Delta x_{i}+\Delta x_{i-1}\right)} \Psi_{i+1, j}^{(n)} \\
& -j \frac{\Delta z}{2 n_{0} k \Delta x_{i-1}\left(\Delta x_{i}+\Delta x_{i-1}\right)} \Psi_{i-1, j}^{(n)} \\
& -j \frac{\Delta z}{2 n_{o} k \Delta y_{j}\left(\Delta y_{j}+\Delta y_{j-1}\right)} \Psi_{i, j+1 l}^{(n)} \\
& -j \frac{\Delta z}{2 n_{o} k \Delta y j-1(\Delta y j+\Delta y j-1)} \Psi_{i, j-1}^{(n)} \\
& +\left\{j \frac{\Delta z}{2 n_{0} k\left(\Delta x_{i}+\Delta x_{i-1}\right)}\left(\frac{1}{\Delta x_{i}}+\frac{1}{\Delta x_{i-1}}\right)\right. \\
& +j \frac{\Delta z}{2 n_{0} k\left(\Delta k_{j}+\Delta y_{j-1}\right)}\left(\frac{1}{\Delta y_{j}}+\frac{1}{\Delta y_{j-1}}\right) \\
& \left.-j \frac{\Delta z k\left(n_{i, j}^{2}-n_{0}^{2}\right)}{4 n_{0}}-1\right\} \Psi_{i, j}^{(n)} \\
& =j \frac{\Delta z}{2 n_{0} k \Delta x_{i}\left(\Delta x_{i}+\Delta x_{i-1}\right)} \Psi_{i+1, j}^{(n-1)} \\
& +j \frac{\Delta z}{2 n_{0} k \Delta x_{i-1}\left(\Delta x_{i}+\Delta x_{i-1}\right)} \Psi_{i-1, j}^{(n-1)} \\
& +j \frac{\Delta z}{2 n_{0} k \Delta y_{j}\left(\Delta y_{j}+\Delta y_{j-1}\right)} \Psi_{i, j+1}^{(n-1)} \\
& +j \frac{\Delta z}{2 n_{0} k \Delta y_{j-1}\left(\Delta y_{j}+\Delta y_{j-1}\right)} \Psi_{i, j-1}^{(n-1)}
\end{aligned}
$$

$$
\begin{aligned}
& -\left\{j \frac{\Delta z}{2 n_{0} k\left(\Delta x_{i}+\Delta x_{i-1}\right)}\left(\frac{1}{\Delta x_{i}}+\frac{1}{\Delta x_{i-1}}\right)\right. \\
& +j \frac{\Delta z}{2 n_{0} k\left(\Delta y_{j}+\Delta y_{j-1}\right)}\left(\frac{1}{\Delta y_{j}}+\frac{1}{\Delta y_{j-1}}\right) \\
& \left.-j \frac{\Delta z k\left(n_{i, j}^{2}-n_{0}^{2}\right)}{4 n_{0}}+1\right\} \Psi_{i, j}^{(n-1)}
\end{aligned}
$$

where $\Delta x_{i}=x_{i+1}-x_{i}$, and $\Delta y_{j}=y_{j+1}-y_{j}$. As (1) represents a set of implicit equations it must be solved iteratively. (1) is of course also valid for a uniform mesh. If the sample points $\left(x_{i}^{(n-1)}, y_{j}^{(n-1)}\right)$ and $\left(x_{i}^{(n), y_{j}(n)}\right)$ are not equal (1) may not be used directly. We solve this problem by calculating a new set of sampled field values $\theta_{i, j}^{(n-1)}$ at the points $\left(x_{i}^{(n)}, y_{j}^{(n)}\right.$ by parabolic interpolation of the field points $\Psi_{i, j}^{(n-1)}$. As long as the number of sample points inside the largest waveguide core was greater than 100 for 3D and 10 for 2D we observed no instabilities due to this interpolation.

\section{NONUNIFORM MESH FOR STEP-INDEX WAVEGUIDES}

In the following we show calculations for step index waveguides as an example but the developed FDBPM may be used for any refractive index distribution. Fig. 1 shows the mesh that is used. The minimum distances between two mesh points in the $x$-direction $\Delta x_{\min }$ and $y$-direction $\Delta y_{\min }$ and the increments $\delta_{x}$ and $\delta_{y}$, respectively, are found from the following equations:

$$
\begin{gathered}
\delta_{x}=(1.5)^{1 / i \max }-1 \quad \delta_{y}=(1.5)^{1 / j \max }-1 \\
\Delta x_{\min }=\delta_{x} a_{r} \quad \Delta y_{\min }=\delta_{y} b_{s}
\end{gathered}
$$

where $a_{r}$ is the largest core width (waveguide number $r$ ) and $b_{s}$ is the largest core height (waveguide number $s$ ). $2 i_{\max }-1$ and $2 j_{\max }-1$ are the number of mesh points inside the largest core width and core height, respectively. For mesh points at the core-cladding interface we define the refractive index to be the mean value of the core and cladding index. This means that we may use (1) at all points. At the boundaries of the computational window we use $\Psi=0$ as the window can be chosen large due to the nonuniform mesh. More sophisticated boundary conditions may of course be incorporated [2].

\section{ACCURACY AND TIME CONSUMPTION}

In Fig. 2 the coupling length $L$, defined on the inset of the figure, has been calculated for a free space wavelength of $1.55 \mu \mathrm{m}$ by the four approaches described above. The waveguides have cross sections of $6.6 \mu \mathrm{m}^{2}$, and refractive index differences of 0.01 . The other geometrical data are shown on the inset on Fig. 2. $P_{1}$ represents the input power and $P_{1}^{\prime}$ and $P_{2}^{\prime}$ are the output powers. The coupler is chosen so that all calculations could be made on a computer workstation when the mesh size was chosen to be $300 \cdot 300 \mu \mathrm{m}^{2}$. The step length $\Delta z$ was chosen to 10 $\mu \mathrm{m}$ for the smallest meshes and $0.1 \mu \mathrm{m}$ for the largest. The 2D-methods converge to $L=900 \mu \mathrm{m}$ while the 


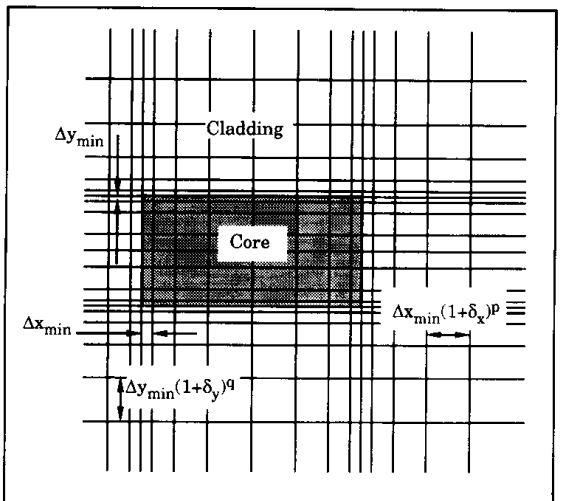

Fig. 1. Nonuniform mesh near a rectangular core cross-section waveguide.

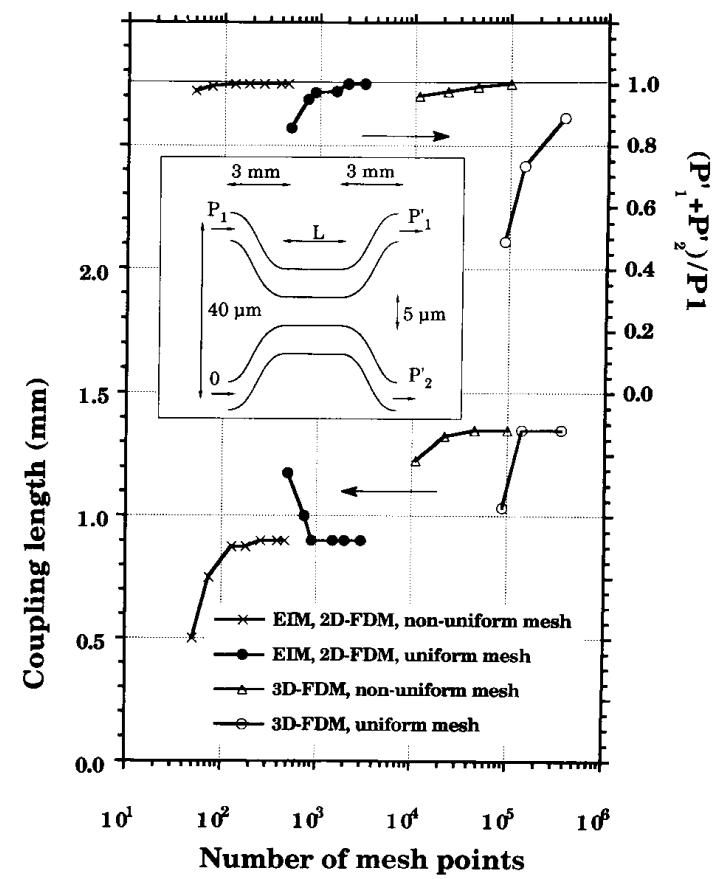

Fig. 2. Coupling length (lower curves) and relative power remaining in the waveguide modes after propagation through the coupler (upper curves) as functions of the number of mesh points. The coupler is defined on the inset.

3D-methods converge to $L=1350 \mu \mathrm{m}$. So, for this particular coupler the reduction of the original 3D-problem to a 2D-problem by the use of the effective index method introduces an error of around $30 \%$. Since the coupler is long enough to assure negligible bending losses we have ideally $P_{1}^{\prime}+P_{2}^{\prime}=P_{1}$. $\left(P_{1}^{\prime}+P_{2}^{\prime}\right) / P_{1}$ is also shown on Fig. 2. For the $2 \mathrm{D}$-methods around 200 points are needed for the nonuniform mesh and 800 for the uniform mesh. For the 3D-methods the figures are 22000 and 360000 for the nonuniform and the uniform mesh, respectively, when $\left(P_{1}^{\prime}+P_{2}^{\prime}\right) / P_{1} \geq 90 \%$ is required. This means that using a

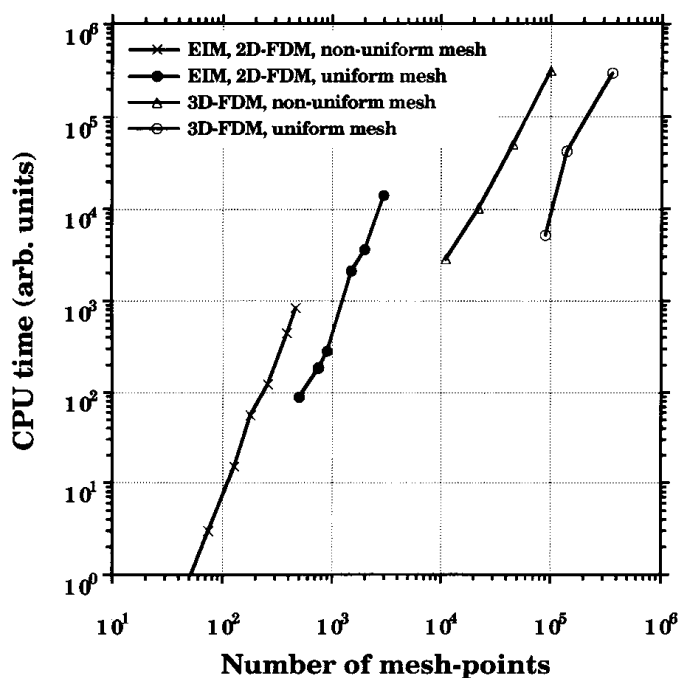

Fig. 3. CPU-time for the four FDBPM-approaches described in the text.

nonuniform mesh as compared to using a uniform mesh gives a reduction of the needed number of mesh points of 4 in the $2 \mathrm{D}$ and 16 in the 3D case. We have also compared the CPU times for the four approaches as shown in Fig. 3. As seen does the time consumption approximate a power dependence on the number of mesh points for all four approaches, indicating that the calculation time due to the iterations increases linearly with the number of mesh points. For the $2 \mathrm{D}$ approach one gains a factor of approximately 3 in time consumption by using a nonuniform mesh instead of a uniform mesh. For the $3 \mathrm{D}$ approach one gains a factor of approximately 30 .

\section{CONCLUSION}

A finite difference method using a nonuniform $z$ varying mesh has been developed. The method is used to calculate the coupling length for a rectangular core cross section, step index single mode waveguide $3 \mathrm{~dB}$ coupler with curved end-sections. Comparing the results with results obtained with a standard finite difference method we found that the computation time needed for a required accuracy is reduced by a factor of approximately 3 for $2 \mathrm{D}$ and 30 for 3D-approaches.

\section{ACKNOWLEDGMENT}

The authors wish to thank Dr. E. Nicolaisen for fruitful discussions.

\section{REFERENCES}

[1] M. D. Feit and J. A. Fleck, "Light propagation in graded-index optical fibers," Appl. Opt., vol. 17, no. 24, pp. 3990-3998, 1978.

[2] R. Accornero, M. Artiglia, G. Coppa, P. Di Vita, G. Lapenta, M Potenza, and P. Ravetto, "Finite Difference method for the analysis of integrated optical waveguides," Electron. Lett., vol. 26, no. 23, pp. $1959-1960,1990$.

[3] S. Chu and S. K. Chaudhuri, "A finite-difference time-domain method for the design and analysis of guided-wave optical structures," J. Lightwave Technol., vol. 7, pp. 2033-2038, Dec. 1989. 
[4] D. Yevick and B. Hermansson, "Split-step finite difference analysis of rib waveguides," Electron. Lett., vol. 25, no. 7, pp. 461-462, 1989.

[5] D. Yevick, J. Yu, M. Munowitz, and D. Vezzetti, "Modal analyses of semiconductor rib waveguides employing nonequidistant grids," J. Opt. Soc. Amer. A, vol. 8, no. 9, pp. 1385-1388, 1991.

[6] R. M. Knox and P. P. Toulios, in Proc. MRSI Symp. Submillimiter Waves, J. Fox, Ed., Polytechnic Press, Brooklyn, NY, 1970.

\title{
Numerically Stable Formulation of the Transverse Resonance Method for Vector Mode-Field Calculations in Dielectric Waveguides
}

\author{
Aasmund Sv Sudbø
}

\begin{abstract}
A new formulation of the eigenvalue problem obtained with the old mode matching or transverse resonance method for mode-field calculations in dielectric waveguides is presented. The formulation is numerically stable for a large class of waveguides of practical importance, and vector mode fields may be calculated much faster and more accurately than with sophisticated methods like finite element methods.
\end{abstract}

$\mathrm{O}$ $\mathrm{NE}$ of the oldest numerical techniques used for calculation of mode fields in dielectric waveguides is that of mode matching (MM) [1]-[4], also developed for ridge waveguides as the transverse resonance method or the equivalent network method [4]-[7]. The main attraction of the method is that it is straightforward. The full vector modes of dielectric waveguides can be computed with the method, and semivectorial and scalar approximations emerge in a particularly transparent manner, as does also the widely used effective index approximation [3].

For very simple waveguide geometries like rectangular guides or symmetric rib guides the method is known to produce accurate mode field distributions with very small computational effort [4], [5]. The method has a reputation for not living up to its promises when generalized to more practical geometries. The cause of this reputation and how to obtain a formulation that delivers as promised for general waveguide cross sections is the subject of this contribution. Only the essential final equations are presented here, and the reader is referred to [8] for details. The equivalent network analogy is of no use in arriving at

Manuscript received November 11, 1992.

The author is with the Norwegian Telecom Research, N-2007 Kjeller, Norway and the Department of Physics, University of Oslo, N-0316 Oslo, Norway.

IEEE Log Number 9207608. the formulation, thus "mode matching" is a more appropriate term than "transverse resonance" for describing the method.

First, let us talk about notation. We consider the waveguide to be a sandwich of "slices" numbered $m=$ $1,2 \ldots M$. Each slice is considered to be cut from a film waveguide with film layers numbered $n=1,2 \ldots N$. It is therefore meaningful to use the label $m$ for the whole film waveguide that slice no. $m$ is cut from as well. Layer no. $n$ in slice or film no. $m$ has index of refraction $n^{(m, n)}$ and relative permittivity $\epsilon^{(m, n)}=n^{(m, n) 2}$. We choose our $x$-axis parallel to the film layers and perpendicular to the slice interfaces, our $y$-axis perpendicular to the film layers and parallel to the film slice interfaces, and our $z$-axis along the waveguide, parallel to the layers and slices. Let $c$ be the speed of light, $\omega$ the angular frequency, $\lambda_{o}$ the corresponding wavelength, and $k_{o}$ the corresponding angular repetency in vacuum, so that $k_{o}=\omega / c=2 \pi / \lambda_{o}$. Let the thickness of layer $n$ be $d_{y}^{(n)}$ and the position of the interface between layer $n-1$ and $n$ be $y^{(n)}$, so that $d_{y}^{(n)}=y^{(n+1)}-y^{(n)}$. Similarly, let the thickness of film slice $m$ be $d_{x}^{(m)}$ and the slice interface positions be at $x^{(m)}$, so that $d_{x}^{(m)}=x^{(m+1)}-x^{(m)}$. The relative permittivity in film $m$ is $\epsilon^{(m)}(y)=\epsilon^{(m, n)}$ for $y^{(n)}<y<y^{(n+1)}$.

The waveguide mode field in slice no. $m$ is expanded in TE- and TM-modes (or just scalar modes if the guide is weak) of the corresponding film, and each mode field component can be expressed as exp $\left[i\left(\omega t-k_{z} z\right)\right]$ times

$$
\begin{aligned}
F^{(m)}(x, y)= & \sum_{p=e, h} \sum_{k=1}^{\infty}\left[u_{p k}^{(m)}(x) F_{s p k}^{(m)}(y)\right. \\
& \left.+\dot{u}_{p k}^{(m)}(x) F_{a p k}^{(m)}(y)\right]
\end{aligned}
$$

where the film mode amplitudes $u_{p k}^{(m)}(x)$ have $x$-deriva- 